\title{
Semi-Definite Relaxations for Minimum Bandwidth and other Vertex-Ordering problems.
}

\author{
Avrim Blum $^{\dagger} \quad$ Goran Konjevod ${ }^{\ddagger} \quad$ R. Ravi ${ }^{\S} \quad$ Santosh Vempala
}

November 13, 1998

\begin{abstract}
We present simple semi-definite programming relaxations for the NP-hard minimum bandwidth and minimum length linear ordering problems. We then show how these relaxations can be rounded in a natural way (via random projection) to obtain approximation guarantees for both of these vertex-ordering problems.
\end{abstract}

\section{Introduction}

Let the vertices of an undirected graph be ordered $1,2, \ldots, n$. We define the dilation of an edge $(i, j)$ as the difference $|i-j|$, i.e., the length of the edge when the vertices of the graph are placed on the line in the order $1,2, \ldots, n$.

Given an undirected graph $G=(V, E)$, we consider the following two problems:

1. Minimum Bandwidth: find an ordering that minimizes the maximum dilation among all the edges, i.e., minimizes

$$
\max _{e \in E} \operatorname{dilation}(e) \text {. }
$$

2. Minimum-length Linear Ordering: find an ordering that minimizes the length (the $L_{2}$ norm) of the ordering, which is defined as:

$$
\sqrt{\sum_{e \in E} \operatorname{dilation}(e)^{2}} .
$$

That is, the squared length is the sum of the squares of dilations of the edges.

*A preliminary version of this paper appeared as "Semi-Definite Relaxations for Minimum Bandwidth and other Vertex-Ordering problems", in Proceedings of the 30th Annual ACM Symposium on Theory of Computing, 90-99, 1998.

${ }^{\dagger}$ School of Computer Science, Carnegie Mellon University, Pittsburgh, PA 15213-3890. Supported in part by NSF National Young Investigator grant CCR-9357793. Email: avrimecs.cmu.edu

${ }^{\ddagger}$ Dept. of Math. Sciences, Carnegie Mellon University, Pittsburgh, PA 15213-3890. Supported by an NSF CAREER grant CCR-9625297. Email: konjevodeandrew.cmu.edu

${ }^{\S}$ GSIA, Carnegie Mellon University, Pittsburgh, PA 15213-3890. Supported by an NSF CAREER grant CCR-9625297. Email: ravi@cmu.edu

IDepartment of Mathematics, Massachusetts Institute of Technology, Cambridge, MA 02139. Part of this work was done while the author was in the School of Computer Science, Carnegie Mellon University, Pittsburgh, PA 15213-3890. Email: vempala@math.mit.edu 
We present new approximation algorithms for both these problems. Our main algorithmic tool is semi-definite programming. Using a simple semi-definite relaxation we derive an $O\left(\sqrt{\frac{n}{b}} \log n\right)$ approximation for the minimum bandwidth on an $n$-node graph with bandwidth $b$. A refinement of this relaxation allows us to get an $O\left(\log ^{2} n\right)$ approximation for the minimum-length linear ordering problem ${ }^{1}$.

Recently (and independently) Feige [6] introduced the notion of a volume-respecting embedding of an undirected graph, and used it to achieve a poly-logarithmic approximation for the bandwidth problem. Interestingly there are many similarities between the two approaches. Specifically, the rounding procedure of our algorithm, projection to a random line, is also a key step in his algorithm. Our relaxation for the minimum-length ordering problem was developed after Feige's results were announced, and was inspired by his work.

Early interest in these kinds of ordering problems in the 1950 's was fueled by research in the area of solvers for sparse symmetric linear systems of equations, using Gaussian elimination (such as in the finite element analysis of steel frameworks). As a heuristic to minimize the space, time and total work in the elimination procedure, it is desirable to reorder the rows (and columns) of the matrix so as to collect all the non-zero entries within a band of small width centered at the diagonal. When the (symmetric) non-zero elements of the matrix are viewed as vertex adjacencies in an undirected graph, then the reordering problem is the minimum bandwidth problem on this graph. Another application of bandwidth minimization is in search algorithms, where the minimum-bandwidth ordering of the vertices of the problem's constraint graph can be used as a branching order that reduces backtracking. For a survey on the bandwidth problem and early approaches, see [2].

The minimum bandwidth problem was first shown to be NP-hard in 1976 [15], and later even for trees of degree at most three and for caterpillars $[7,13]$. Approximations algorithms have been known only for some special families of graphs, such as caterpillars or asteroidal triple-free graphs $[11,12]$.

\section{The Semi-Definite Relaxation}

Our approximation algorithm begins with an SDP (Semi-Definite Programming) relaxation. First we motivate and describe the relaxation for the bandwidth problem and then the relaxation for the minimum-length linear ordering problem.

Semidefinite programming is the optimization of a linear function over variables drawn from a symmetric matrix subject to linear constraints on these variables and the added requirement that this symmetric matrix be positive semidefinite. Semidefinite programs can be solved to within an arbitrarily small additive error in polynomial-time [8] - for more background, see the description in [9]. Our semidefinite relaxation for the bandwidth problem is inspired by the pioneering work of Goemans and Williamson [9] on the Maximum Cut problem, and is based on the following observation that their work motivates and uses: An $n \times n$ symmetric positive semidefinite matrix $A$ can be decomposed into $A=B^{T} B$ where $B$ is an $m \times n$ matrix of full row rank (hence $m \leq n$ ) via an incomplete Cholesky factorization (see, e.g., [10]). Moreover, if we insist that $a_{i i}=d$ for all $i$, then the matrix $A$ corresponds via the decomposition to a set of equal-length vectors $b_{1}, b_{2}, \ldots, b_{n}$ in $\boldsymbol{R}^{m}$

\footnotetext{
${ }^{1}$ The preliminary version of this paper [1] erroneously claimed an $O\left(\log ^{\frac{3}{2}} n\right)$-approximation ratio for this problem. We thank Seffi Naor [16] for bringing the error in the analysis to our attention.
} 
(each of Euclidean length $\sqrt{d}$ ), namely, $b_{i}$ is the $i^{\text {th }}$ column of $B$, where $a_{i j}=b_{i} \cdot b_{j}$ (the dot product of $b_{i}$ and $b_{j}$ ). This allows us to view the solution to a SDP equivalently as a set of vectors in $\boldsymbol{R}^{m}$ for some $m \leq n$, obeying some extra linear constraints that we stipulate on the dot products of these vectors.

\subsection{Bandwidth}

The minimum bandwidth problem on an undirected graph $G=(V, E)$ is equivalent to the following geometrical embedding problem: Assign the nodes of the graph to distinct equispaced points along a quarter-circle of radius $n$ in the positive quadrant of a 2-dimensional plane, such that the maximum value of the Euclidean length of any edge of the graph is minimized. The projection of this ordering on either of the co-ordinate axes bounding this quadrant recovers the optimal bandwidth ordering since the objective functions (Euclidean distance in the quarter-circle and linear distance in the line) are monotonically related. The following is then a non-convex quadratic programming formulation for the bandwidth problem. We use $\hat{x}$ and $\hat{y}$ to denote the basis vectors along the $x$ and $y$ co-ordinates respectively. Also $|v|$ is used to denote the Euclidean length of a vector $v$.

$$
\begin{aligned}
\operatorname{minimize} & b \\
\left|v_{i}\right| & =n \quad \forall i \in\{1, \ldots, n\} \\
\left|v_{i}-v_{j}\right| & \leq b \quad \forall(i, j) \in E
\end{aligned}
$$

and the $v_{i}$ 's are two-dimensional vectors each distinctly assigned to a point of the form $n \cos \left(\frac{\pi \jmath}{2 n}\right) \hat{x}+n \sin \left(\frac{\pi \jmath}{2 n}\right) \hat{y}$ for some positive $j$.

The last set of constraints cannot be enforced if we wish to carry out the optimization in polynomial time, so we relax the dimensionality of the vectors $v_{i}$ and add more constraints to strengthen the resulting program. The extra constraints we add are "spreading" constraints, in the spirit of Even, Naor, Rao and Schieber [5]. With only constraints such as (2) and (3), the program will simply produce one single vector as its solution to all the $v_{i}$. We want instead that the vectors be spread out as in a line. For instance, on a line, for any point $p$ there are at most $2 k$ other points within distance $k$ of $p$. We add some new constraints $((8)$ in the formulation below) to enforce this condition. We finally arrive at the following semidefinite programming formulation,

$$
\begin{aligned}
\text { minimize } & b \\
v_{i} \cdot v_{j} & \geq 0 \quad \forall i, j \in\{1, \ldots, n\} \\
\left|v_{i}\right| & =n \quad \forall i \in\{1, \ldots, n\} \\
\left|v_{i}-v_{j}\right| & \leq b \quad \forall(i, j) \in E \\
\frac{1}{|S|} \sum_{j \in S}\left(v_{i}-v_{j}\right)^{2} & \geq \frac{1}{6}\left(\frac{|S|}{2}+1\right)(|S|+1) \quad \forall S \subseteq\{1, \ldots, n\}, \forall i \in\{1, \ldots, n\}
\end{aligned}
$$

The goal of the above constraints is to enforce a near-linear embedding of the vertices while minimizing the value of $b$, which is the maximum dilation of any edge in the relaxation. 
Formally, constraint set (7) states that for any edge in the graph, the distance between the corresponding vectors should be at most the optimal bandwidth. From our discussion above, it is perhaps easier to see that $(7)$ is a legal SDP constraint if we rewrite it as $\left(v_{i}-v_{j}\right) \cdot\left(v_{i}-v_{j}\right) \leq b^{2}$.

Constraints (5) are primarily for ease of analysis. Constraints (8) are the spreading constraints motivated earlier. These constraints are useful in the analysis of the rounding algorithm where we bound the probability that two given points in the vector representation of the solution fall into an interval of fixed width in a random projection to a line (i.e., when all the points are projected to their dot product with a random unit vector ${ }^{2}$ passing through the origin). This probability is inversely proportional to the distance between the points (Lemma 5) and the spreading constraints allow us to upper bound this probability effectively.

Although there are exponentially many constraints in (8), it is not hard to construct a separation oracle for them, and hence the SDP can be solved in polynomial time (see Grötschel, Lovász, Schrijver [8]). To answer the separation problem for (8) for a given node $i$, simply sort the vertices $j \neq i$ in increasing order of $\left(v_{i}-v_{j}\right)^{2}$ and check for violation each of the $n-1$ sets that occur as prefixes in this order. It is easy to see that if any set $S$ violates (8) for vertex $i$, then the prefix of vertices in this order of size $|S|$ also violates (8) for $i$. This is also the same separation oracle used by Even et al. [5] in their work on spreading metrics.

Let us refer to the above formulation as the bandwidth SDP. Suppose $b^{*}$ is the optimal bandwidth. Then by lifting the optimal bandwidth ordering to the equi-spaced embedding in the quarter-circle described above, it is easy to verify that all the constraints are satisfied to give an objective function value of at most $\frac{\pi}{2} b^{*}$.

\section{$2.2 \quad$ Minimum-length ordering}

The SDP formulation for the Minimum-length ordering problem is similar to the bandwidth SDP. Technically, the analysis in this case will require bounding the probability that three points fall into a fixed width interval in a random projection (instead of just two points as in the bandwidth analysis). This probability can be shown to be inversely proportional to the area of the triangle formed by the three points (Lemma 6). For this reason, we add constraints in this case that lower bound the areas of triangles formed by points in the vector representation of the solution. Specifically, if we let $A(i, j, k)$ denote the area of the triangle formed by $v_{i}, v_{j}, v_{k}$, our SDP relaxation of the minimum-length linear ordering problem is the following. (The fact that it is indeed a relaxation will be established in Lemma 8.)

$$
\begin{aligned}
\text { minimize } & \sum_{(i, j) \in E}\left(v_{i}-v_{j}\right)^{2} \\
v_{i} \cdot v_{j} & \geq 0 \quad \forall i, j \in\{1, \ldots, n\} \\
\frac{1}{|S|} \sum_{j \in S}\left(v_{i}-v_{j}\right)^{2} & \geq \frac{1}{6}\left(\frac{|S|}{2}+1\right)(|S|+1) \quad \forall S \subseteq\{1, \ldots, n\}, \forall i \in\{1, \ldots, n\}
\end{aligned}
$$

\footnotetext{
${ }^{2}$ Here and henceforth, we use the term "unit vector" to denote a vector of unit length, not only those along the co-ordinate axes.
} 


$$
\frac{1}{|S|} \sum_{k \in S} A^{2}(i, j, k) \geq \epsilon\left|v_{i}-v_{j}\right|^{2}|S|^{2} \quad \forall S \subseteq\{1, \ldots, n\}, \forall i, j \in\{1, \ldots, n\}
$$

The first two sets of constraints are identical to (5), (8) above. Instead of constraining the length of each individual edge as in (7), we minimize the squared length of the ordering (sum of squares of edge lengths). This is a linear function of the $v_{i} \cdot v_{j}$. The constraint set (9) was motivated earlier and will be useful in the analysis; $\epsilon$ is a constant greater than 0 that can be calculated from Lemma 11 in the Appendix. We address below the incorporation of constraints (9) in the semidefinite formulation.

Fact 1 For two vectors $v_{i}, v_{j}$, the square of the area of the triangle they form with the origin is given by

$$
\frac{1}{4}\left|\begin{array}{cc}
v_{i} \cdot v_{i} & v_{i} \cdot v_{j} \\
v_{i} \cdot v_{j} & v_{j} \cdot v_{j}
\end{array}\right|
$$

Hence, for any three vectors, $v_{i}, v_{j}, v_{k}$, the area $A(i, j, k)$ of the triangle formed by them, which is the same as the area of the triangle formed by $v_{j}-v_{i}, v_{k}-v_{i}$ and the origin, can be computed using

$$
A^{2}(i, j, k)=\frac{1}{4}\left|\begin{array}{cc}
\left(v_{j}-v_{i}\right) \cdot\left(v_{j}-v_{i}\right) & \left(v_{j}-v_{i}\right) \cdot\left(v_{k}-v_{i}\right) \\
\left(v_{j}-v_{i}\right) \cdot\left(v_{k}-v_{i}\right) & \left(v_{k}-v_{i}\right) \cdot\left(v_{k}-v_{i}\right)
\end{array}\right| .
$$

Further, the constraint, $A^{2}(i, j, k) \geq c$ for a real number $c$ is a convex constraint. Note that for an arbitrary matrix $X$, the constraint $D E T(X) \geq c$ may not be convex; however when $X$ is restricted to being positive semi-definite (as in our case), it becomes convex. (See, e.g., [14], pp. 239.)

\section{The Algorithm}

Given an undirected graph $G=(V, E)$, the approximation algorithm for both problems is as follows. The only difference between the two problems is in the SDP formulations.

1. Solve the $S D P$ relaxation for $G$. Let the solution obtained be $v_{1}, \ldots, v_{n}$.

2. Pick a random line through the origin, i.e., a random unit vector $\ell$.

3. Project $v_{1}, \ldots, v_{n}$ on to the line $\ell$.

4. Output the vertex-ordering along this line, i.e., in increasing values of $v_{i} \cdot \ell$.

We show that the algorithm with the bandwidth SDP finds an ordering of bandwidth at most $O\left(\sqrt{\frac{n}{b}} \log n\right)$ times the optimum with high probability. For the minimum-length ordering problem we will show that this algorithm gives an ordering of length at most $O\left(\log ^{2} n\right)$ times the optimum, with high probability. 


\subsection{Overview of Bandwidth analysis}

The outline of the analysis for the Bandwidth performance guarantee is as follows. Imagine slicing up the ball of radius $n$ into strips orthogonal to $\ell$ of width $b / \sqrt{n}$. The first claim is that with high probability, no edge in $G$ crosses more than $O(\sqrt{\log n})$ strips. The reason is that for any edge $(i, j)$ we have $\left|v_{i}-v_{j}\right| \leq b$ (by constraint 7 ) and since $\ell$ was chosen randomly, with high probability we have $\left|\left(v_{i}-v_{j}\right) \cdot \ell\right| \leq c\left|v_{i}-v_{j}\right| \sqrt{\log n} / \sqrt{n}$ (i.e., the vector $v_{i}-v_{j}$ is "nearly orthogonal" to the line $\ell$; see Lemma 3$)$. So, to prove an $\tilde{O}(\sqrt{n})$ approximation for the minimum bandwidth it suffices to prove that with "reasonable" probability, every strip has at most $\tilde{O}(\sqrt{n})$ points inside.

For a given strip $s$ (say, the strip corresponding to the interval $[i b / \sqrt{n},(i+1) b / \sqrt{n}]$ on line $\ell$ ), the probability over the choice of $\ell$ that a given point $v \in G$ falls into $s$ is at most $O(b / n)$. (This is because there are $O(n \sqrt{n} / b)$ strips total, and the middle $n / b$ of them roughly equally divide up most of the probability.) Thus, the expected number of points in any given strip is only $O(b)$.

What about the variance? To calculate this we need to upper-bound the probability that a given pair of points $v_{i}, v_{j}$ both fall into a given strip $s$. This is roughly equal to $\operatorname{Pr}\left[v_{i}\right.$ falls into $\left.s\right] \cdot \operatorname{Pr}\left[\left|v_{i}-v_{j}\right| \cdot \ell \leq b / \sqrt{n}\right]$. The first quantity, as described above, is $O(b / n)$, while the latter quantity is $O(b / d)$ if $\left|v_{i}-v_{j}\right| \leq d$. At this point, we use constraints (8) to show that there cannot be too many pairs of points $v_{i}, v_{j}$ that are too close together. This allows us to bound the variance which then yields the final results. For slightly improved bounds, we use strips of width $\Theta(b \sqrt{\log n} / \sqrt{n})$ instead of $b / \sqrt{n}$.

\subsection{Overview of Minimum-length Ordering analysis}

The analysis is very similar to the analysis of the bandwidth guarantee. The first step is to show that the optimal ordering can be turned into a solution for the SDP formulation without much worsening in the objective function. We do this in Lemma 8 by using a lifted embedding of the optimal solution in $\log n$-dimensions so as to obey the triangle lower-bound constraints.

The proof of the rounding guarantee relies on bounding the expected value of the different terms in the objective function, one for each edge $(i, j)$ in the graph $G$. This term for the edge $(i, j)$ is the square of the number of points that fall between $i$ and $j$ in the random projection. Algebraic simplification shows that what is required to be bounded is the probability that a pair of other points $k$ and $l$ both fall between $i$ and $j$ in the random projection. As before, since $\ell$ was chosen randomly, with high probability we have $\left|\left(v_{i}-v_{j}\right) \cdot \ell\right| \leq c\left|v_{i}-v_{j}\right| \sqrt{\log n} / \sqrt{n}$, and so we are left to bound the probability that the three vectors $v_{i}, v_{k}$ and $v_{l}$ all fall in an interval of width $c\left|v_{i}-v_{j}\right| \sqrt{\log n} / \sqrt{n}$. We accomplish this by relating this inversely to the area of the triangle $A(i, k, l)$ formed by these vectors and using the lower bounds on the triangle areas.

We present the formal analyses in the next section.

\section{Approximation Guarantees}

We start with a useful lemma about any set of vectors satisfying the constraint set (8). 
Lemma 1 Let $v_{1}, \ldots, v_{n} \in \boldsymbol{R}^{n}$ satisfy the constraint set (8). Then for any ball $B$ of radius $r \geq 1$ in $\boldsymbol{R}^{n}$ (not necessarily centered at the origin)

$$
\left|B \cap\left\{v_{1}, \ldots, v_{n}\right\}\right| \leq O(r) .
$$

Proof. Let $S=\left\{j: v_{j} \in B\right\}$ and suppose for contradiction that $|S|>7 r$. Pick an arbitrary $i \in S$. By constraint set (8), the average value of $\left(v_{i}-v_{j}\right)^{2}$ over $j \in S$ is at least $\frac{1}{6}(|S| / 2+1)(|S|+1)>\frac{1}{6}(7 r / 2)(7 r)>4 r^{2}$. But, this is clearly impossible because the maximum value of $\left|v_{i}-v_{j}\right|$ is at most the diameter $2 r$.

Next, we make a few observations regarding random projections.

Lemma 2 Let $v_{1}, v_{2}, v_{3} \in \boldsymbol{R}^{n}$. Let $\ell$ be a random unit vector. Let $y_{i}=v_{i} \cdot \ell$. Let $\theta$ be the angle between the vectors $\left(v_{2}-v_{1}\right)$ and $\left(v_{3}-v_{1}\right)$. Then the probability that $y_{1}$ lies between $y_{2}$ and $y_{3}$ is exactly $\theta / \pi$.

Proof. The probability that $v_{1}$ when projected to $\ell$ falls in between the projections of $v_{2}$ and $v_{3}$ is

$$
\operatorname{Pr}\left[v_{2} \cdot \ell \leq v_{1} \cdot \ell \leq v_{3} \cdot \ell\right]+\operatorname{Pr}\left[v_{3} \cdot \ell \leq v_{1} \cdot \ell \leq v_{2} \cdot \ell\right]
$$

which is the same as

$$
\operatorname{Pr}\left[\left(\left(v_{1}-v_{2}\right) \cdot \ell\right)\left(\left(v_{3}-v_{1}\right) \cdot \ell\right) \geq 0\right]
$$

which is exactly the angle between the vectors $\left(v_{1}-v_{2}\right)$ and $\left(v_{3}-v_{1}\right)$ divided by $\pi$.

Fact 2 The volume of the $n$-dimensional ball of radius $r$ is equal to $\frac{2 r^{n} \pi^{n / 2}}{n \Gamma(n / 2)}$ and its surface area is $\frac{2 r^{n-1} \pi^{n / 2}}{\Gamma(n / 2)}$.

Here $\Gamma(x)$ is Euler's Gamma function; for a positive integer $x, \Gamma(x)=(x-1)$ !.

Lemma 3 Let $v \in \boldsymbol{R}^{n}$. For a random unit vector $\ell$,

$$
\operatorname{Pr}\left[|v \cdot \ell| \leq \frac{c}{\sqrt{n}}|v|\right] \geq 1-e^{-c^{2} / 4}
$$

Proof. The desired probability is the surface of a central band of thickness $2 c / \sqrt{n}$ on a unit sphere, divided by the surface area of the whole sphere. Denote the surface area of the $n$-dimensional sphere of radius $r$ by $A_{n}(r)$. Then the area of the region outside the central band is less than the area of an $n$-dimensional sphere of radius $\sqrt{1-c^{2} / n}$ (since the remaining portions of the unit ball after slicing out the central band is a convex body that can be inscribed in a ball of the smaller radius). Using $A_{n}(r)=K_{n} r^{n-1}$, for $K_{n}$ as in fact 2, we can lower bound the area of the central band as the area of the unit sphere minus the area of a sphere of radius $\sqrt{1-c^{2} / n}$. Thus the desired probability is at least

$$
\begin{aligned}
\frac{A_{n}(1)-A_{n}\left(\sqrt{1-\frac{c^{2}}{n}}\right)}{A_{n}(1)} & =1-\left(1-\frac{c^{2}}{n}\right)^{n / 2} \\
& \geq 1-e^{-c^{2} / 4}
\end{aligned}
$$


Lemma 4 Let $v \in \boldsymbol{R}^{n}$. For a random unit vector $\ell$,

$$
\operatorname{Pr}\left[|v \cdot \ell| \leq \frac{1}{c \sqrt{n}}|v|\right]=O\left(\frac{1}{c}\right)
$$

Proof. The desired probability can be upper-bounded by $4 / c \sqrt{n}$ times the surface area of the $(n-1)$-dimensional unit ball, divided by the surface area of the $n$-dimensional unit ball. The factor of 4 is due to loosely upper-bounding the curvature of the $n$-dimensional ball within a width of $1 / c \sqrt{n}$ in both directions above and below the origin. This is at most

$$
\frac{4 A_{n-1}}{c \sqrt{n} A_{n}} \leq \frac{4}{c \sqrt{n \pi}} \frac{\Gamma\left(\frac{n+1}{2}\right)}{\Gamma\left(\frac{n}{2}\right)} \leq O\left(\frac{1}{c}\right)
$$

We consider the following event: two points $x, y$ on the surface of the ball of radius $n$, at a distance $d$ from each other are projected on to a random line. What is the probability that $x$ and $y$ fall in any fixed interval of width $W$ on the line? The following lemma, crucial to our analysis, bounds this probability.

Lemma 5 Let $x, y$ be arbitrary vectors of length $n$ in $\boldsymbol{R}^{n}$ such that $|x-y|=d$ and $x \cdot y \geq 0$. Let $\ell$ be a random unit vector. Then, for any fixed $\alpha$ and width $W$,

$$
\operatorname{Pr}[\alpha \leq x \cdot \ell, y \cdot \ell \leq \alpha+W]=O\left(\frac{W^{2}}{d}\right)
$$

Proof. For convenience, rotate the sphere so that

$$
x=\left(-d / 2, \sqrt{n^{2}-d^{2} / 4}, 0, \ldots\right)
$$

and

$$
y=\left(d / 2, \sqrt{n^{2}-d^{2} / 4}, 0, \ldots\right) .
$$

Let vector $v=y-x=(d, 0, \ldots)$, and let $\ell=\left(\ell_{1}, \ell_{2}, \ldots\right)$ be our randomly chosen unit vector. Note that in order for the event in question to occur, it must be the case that $|v \cdot \ell| \leq W$. Therefore,

$$
\operatorname{Pr}[\alpha \leq x \cdot \ell, y \cdot \ell \leq \alpha+W] \leq \operatorname{Pr}[|v \cdot \ell| \leq W] \cdot \operatorname{Pr}[\alpha \leq x \cdot \ell \leq \alpha+W|| v \cdot \ell \mid \leq W] .
$$

Since $|v \cdot \ell|=\left|\ell_{1}\right| \cdot d$, we have $\operatorname{Pr}[|v \cdot \ell| \leq W]=\operatorname{Pr}\left[\left|\ell_{1}\right| \leq W / d\right]$, which is $O(W \sqrt{n} / d)$ by Lemma 4.

Given the event that $\left|\ell_{1}\right| \leq W / d$, the inequality $\alpha \leq x \cdot \ell \leq \alpha+W$ can be relaxed to $\alpha-W / 2 \leq x^{\prime} \cdot \ell^{\prime} \leq \alpha+3 W / 2$, where $x^{\prime}$ and $\ell^{\prime}$ are $n-1$-dimensional vectors consisting of the last $n-1$ components of $x$ and $\ell$. Since $x_{i}=0$ for all $i>2$, this is equivalent to

$$
\alpha-W / 2 \leq \ell_{2} \sqrt{n^{2}-d^{2} / 4} \leq \alpha+3 W / 2 .
$$

The probability of this last event can be upper-bounded by computing the area of the largest possible strip of this width $W$ (the one centered around the equator). By assumption, $x \cdot y \geq 0$, implying that $d \leq n \sqrt{2}$, so $\sqrt{n^{2}-d^{2} / 4} \geq n / \sqrt{2}$. We can now bound the fraction 
of the sphere covered by this strip by $O(W / \sqrt{n})$ as in the proof of Lemma 4 . Thus, we finally get

$$
\begin{aligned}
\operatorname{Pr}[\alpha \leq x \cdot \ell, y \cdot \ell \leq \alpha+W] & =O\left(\frac{W \sqrt{n}}{d} \cdot \frac{W}{\sqrt{n}}\right) \\
& =O\left(\frac{W^{2}}{d}\right) .
\end{aligned}
$$

The following lemma will be useful in the analysis for the minimum length ordering problem.

Lemma 6 Let $v_{1}, v_{2}, v_{3}$ be vectors in $\boldsymbol{R}^{n}$. Then on projection to a random line, the probability that there exists an interval of width $W$ that all three fall into is

$$
O\left(\frac{W^{2} n}{A(1,2,3)}\right)
$$

Proof. Consider the triangle $v_{1} v_{2} v_{3}$. Assume without loss of generality that its smallest angle is the one at $v_{3}$, and that $\left|v_{1}-v_{3}\right| \leq\left|v_{2}-v_{3}\right|$. Notice that the event in question is invariant under translation of the space; thus we may also assume without loss of generality that $v_{3}$ is the origin.

In order for all three points to fall into an interval of width $W$, it must be the case that $v_{1}$ and $v_{2}$ both fall into the interval $[-W, W]$. We bound the probability of the latter event using Lemma 5. Specifically, let $v_{1}^{\prime}=n v_{1} /\left|v_{1}\right|$, let $v_{2}^{\prime}=n v_{2} /\left|v_{2}\right|$, and let $d^{\prime}=\left|v_{2}^{\prime}-v_{1}^{\prime}\right|$. The event that $v_{1}$ and $v_{2}$ both fall into the interval $[-W, W]$ implies the event that $v_{1}^{\prime}$ and $v_{2}^{\prime}$ both fall into the interval $\left[-W n /\left|v_{1}\right|, W n /\left|v_{1}\right|\right]$ since $\left|v_{1}\right| \leq\left|v_{2}\right|$. Since $v_{1}^{\prime}$ and $v_{2}^{\prime}$ are both length $n$ (and $v_{1}^{\prime} \cdot v_{2}^{\prime} \geq 0$ by the assumption that the smallest angle is at $v_{3}$ ), Lemma 5 bounds the probability of this event by

$$
O\left(\frac{W^{2} n^{2}}{\left|v_{1}\right|^{2} d^{\prime}}\right)
$$

Since $v_{3}$ is the smallest angle of the triangle $v_{1} v_{2} v_{3}$, the area of $v_{1} v_{2} v_{3}$ is at most twice the area of $v_{1} v_{2}^{\prime \prime} v_{3}$ where $v_{2}^{\prime \prime}=v_{2}\left|v_{1}\right| /\left|v_{2}\right|$. This area equals $\left(\left|v_{1}\right| / n\right)^{2}$ times the area of $v_{1}^{\prime} v_{2}^{\prime} v_{3}$, and that area is at most $n d^{\prime} / 2$. Thus, $A(1,2,3) \leq\left|v_{1}\right|^{2} d^{\prime} / n$, and the desired probability is

$$
O\left(\frac{W^{2} n^{2}}{\left|v_{1}\right|^{2} d^{\prime}}\right)=O\left(\frac{W^{2} n}{A(1,2,3)}\right) \text {. }
$$

\subsection{Bandwidth}

We begin with the following lemma.

Lemma 7 Suppose $v_{1}, \ldots, v_{n}$ satisfy the constraint set (5), (6), and (8). For a random line $\ell$, let $X$ be the random variable denoting the number of points $v_{i}$ whose projection onto $\ell$ falls into a given interval I of width $W$. Then,

$$
\mathbf{E}[X]=O(W \sqrt{n}) \text { and } \mathbf{E}\left[X^{2}\right]=O\left(W^{2} n \log n\right) .
$$


Proof. Define $X_{i}$ to be the random variable that is 1 if the projection of $v_{i}$ onto $\ell$ falls in $I$ and 0 otherwise. Then from Lemma $4, \mathbf{E}\left[X_{i}\right]=O(W / \sqrt{n})$, which implies

$$
\mathbf{E}[X]=O(W \sqrt{n}) .
$$

Now consider pairs $v_{i}, v_{j}$. By Lemma 5 we have $\mathbf{E}\left[X_{i} X_{j}\right]=O\left(W^{2} / d_{i j}\right)$, where $d_{i j}=\left|v_{i}-v_{j}\right|$. Therefore,

$$
\begin{aligned}
\mathbf{E}\left[X^{2}\right] & =\mathbf{E}\left[\sum X_{i}^{2}+2 \sum X_{i} X_{j}\right] \\
& =O\left(W \sqrt{n}+\sum_{i, j} \frac{W^{2}}{d_{i j}}\right) \\
& =O\left(W^{2} n \log n\right),
\end{aligned}
$$

where the last line follows from Lemma 1, since Lemma 1 implies that for any fixed $i$, $\sum_{j} 1 / d_{i j}=O(\log n)$.

Theorem 1 The algorithm finds an ordering whose bandwidth is at most $O(\sqrt{n / b} \log n)$ times the minimum bandwidth with high probability.

Proof. Let $v_{1}, \ldots, v_{n}$ be the set of vectors of length $n$ found by solving the SDP.

First, using Lemma 3 we have that every edge of $G$, of length at most $b$ in the SDP, when projected down to a random line has length no more than $W=8 b \sqrt{\log n} / \sqrt{n}$ with high probability.

Let $\ell$ be a random line and partition $\ell$ into intervals of width $W$. Using Lemma 3 one more time, with high probability, all vertices on projection fall within the middle $n / b$ intervals (since these have total width $8 \sqrt{n \log n}$ ). Since each edge spans at most two intervals (with high probability), it suffices now just to prove that with reasonable probability, none of these $n / b$ intervals has more that $O(\sqrt{n b} \log n)$ vertices that project into it.

At this point we simply use Lemma 7 . By Lemma 7 , the random variable $X$ denoting the number of vertices that on projection fall into a given interval of width $W$ satisfies $\mathbf{E}\left[X^{2}\right]=O\left(W^{2} n \log n\right)$. Therefore, by Chebychev's inequality

$$
\begin{aligned}
\frac{b}{4 n} & \geq \operatorname{Pr}\left[X>\sqrt{4 n / b} \sqrt{W^{2} n \log n}\right] \\
& =\operatorname{Pr}[X>16 \sqrt{b n} \log n] .
\end{aligned}
$$

Thus, with reasonable probability (3/4), each of the $n / b$ intervals has only $O(\sqrt{n b} \log n)$ vertices that project into it, proving the theorem.

\subsection{Minimum-length ordering}

Let $e=(i, j) \in E$, and upon projection to a random line, let $Y_{i j}$ be the random variable whose value is the dilation of $e$ in the ordering on the line, i.e., the number of points that fall in the span of the edge.

First, we use Lemmas 1 and 2 to show that the expectation of $Y_{i j}$ is at most $\left|v_{i}-v_{j}\right| \log n$ as follows. Let $P$ denote the center of the geodesic joining $v_{i}$ and $v_{j}$, and let $l=\left|v_{i}-v_{j}\right|$. Consider balls with radii $l, 2 l, 4 l, \ldots$ centered at $P$. Let $x_{k}$ be at distance $r l$ from $P$. By 
lemma 2 the probability that $x_{k}$ is projected between $x_{i}$ and $x_{j}$ is at most $\frac{2}{r}$. By lemma 1 , the number of points at a distance of between $r l$ and $2 r l$ from $P$ is $O(r l)$. So the expected number of vertices that fall in between the projections of $x_{i}$ and $x_{j}$ is at most

$$
\sum_{m=1}^{\frac{1}{2} \log n} O\left(2^{m} l\right) \frac{1}{2^{m}}=O(l \log n) .
$$

However we need to bound the second moment, $\mathbf{E}\left(Y_{i j}^{2}\right)$. For this we need to bound the probability that a pair of vertices falls in the span of $e$. Lemma 6 bounds this probability as at most 1 over the area of the triangle formed by the two points and any one of the endpoints of the edge. So, on the whole we would like to ensure that the sum of the inverse areas of the triangles formed by every pair with one endpoint of $e$ is small. This is precisely what the triangle constraints (9) achieve. Just the spreading constraints (8) do not suffice for this.

Below we describe this formally. First we show that the SDP is indeed a near-relaxation (there exists a solution to the SDP with value $\leq O P T \sqrt{\log n}$ ). Then we give the approximation guarantee for the rounding step.

Lemma 8 Let $O P T$ be the value of the minimum length ordering, and $O P T_{S D P}$ be the objective value found by the SDP. Then,

$$
O P T_{S D P}^{2} \leq O P T^{2} \log n
$$

Proof. Without loss of generality, let $0, \ldots, n-1$ be the minimum length ordering of $G$. Let the value of this ordering be OPT, i.e.,

$$
O P T=\sqrt{\sum_{(i, j) \in E}(i-j)^{2}}
$$

We will now construct an embedding of the vertices as vectors $u_{0}, \ldots, u_{n-1} \in \boldsymbol{R}^{\lfloor\log n\rfloor+1}$ such that

$$
\left|u_{i}-u_{j}\right| \leq|i-j| \sqrt{\lfloor\log n\rfloor+1}
$$

and further $u_{0}, \ldots, u_{n-1}$ satisfy the constraints of the minimum length ordering SDP. The lemma follows from these facts.

First, an example. For $n=17$ points, the embedding is:

$(0,0,0,0,0)$

$(6,6,2,2,0)$ 
$(7,7,1,1,1)$

$(8,8,0,0,0)$

$(9,7,1,1,1)$

$(10,6,2,2,0)$

$(13,3,3,1,1)$

$(14,2,2,2,0)$

$(15,1,1,1,1)$

$(16,0,0,0,0)$

The first coordinate is just $i$. The second coordinate is $i$ for $i \leq n / 2$ and $n-i$ after that. The third coordinate goes up to $n / 4$, down to zero, back up to $n / 4$ and back down to zero again. And so on.

In general, let $d$ be the smallest integer such that $2^{d}>n$. Then $i$ is mapped to

$$
\left(i,\left|i \bmod 2^{d-1}-2\left(i \bmod 2^{d-2}\right)\right|, \ldots,\left|i \bmod 2^{d-l+1}-2\left(i \bmod 2^{d-l}\right)\right|, \ldots, i \bmod 2\right) .
$$

That is, the $l^{\text {th }}$ coordinate of $u_{i}$ is $\left|i \bmod 2^{d-l+1}-2\left(i \bmod 2^{d-l}\right)\right|$, for $l=1, \ldots, d$.

Since the $l$ th coordinate of $u_{i}$ differs from the $l$ th coordinate of $u_{j}$ by at most $|i-j|$, we have $\left(u_{i}-u_{j}\right)^{2} \leq d(i-j)^{2}$. So, we have $\left|u_{i}-u_{j}\right| \leq|i-j| \sqrt{\lfloor\log n\rfloor+1}$ as desired. Constraints (8) are satisfied because the construction of the first coordinate ensures that for any $i, j,\left|u_{i}-u_{j}\right| \geq|i-j|$.

Finally, we just need to show that constraints (9) are satisfied. This follows from the fact, given as Lemma 11 in the appendix, that for any $i<j<k$ the area of the triangle formed by $u_{i}, u_{j}, u_{k}$ is $\Omega(|j-i||k-j|)$.

These observations imply that $u_{0}, \ldots, u_{n-1}$ satisfy the SDP, and their objective value is $O(O P T \sqrt{\log n})$.

Let $v_{1}, \ldots, v_{n}$ be the set of vectors found by solving the SDP.

\section{Lemma 9}

$$
\mathbf{E}\left(Y_{i j}^{2}\right)=O\left(\left|v_{i}-v_{j}\right|^{2} \log ^{3} n\right)
$$

Proof. Fix some edge $(i, j)$. Define the random variable $X_{k}$ for each $k=1, \ldots, n$, $k \neq i, j$ to be 1 if on random projection $v_{k}$ is projected in between $v_{i}$ and $v_{j}$ (falls in the span of the edge) and 0 otherwise. Then

$$
Y_{i j}=\sum_{k \neq i, j} X_{k}
$$

and

$$
\begin{aligned}
\mathbf{E}\left(Y_{i j}^{2}\right) & =\sum_{k \neq i, j} \mathbf{E}\left(X_{k}^{2}\right)+\sum_{k, l \neq i, j} \mathbf{E}\left(X_{k} X_{l}\right) \\
& =\sum_{k \neq i, j} \mathbf{E}\left(X_{k}\right)+\sum_{k, l \neq i, j} \operatorname{Pr}[k, l \text { fall between } i, j]
\end{aligned}
$$




$$
\begin{aligned}
& \leq \mathbf{E}\left(Y_{i j}\right)+\sum_{k, l \neq i, j} \operatorname{Pr}\left[k, l, i \text { fall in an interval of width } \frac{4 \sqrt{\log n}}{\sqrt{n}}\left|v_{i}-v_{j}\right|, \text { or } i, j \text { do not }\right] \\
& \left.\leq\left|v_{i}-v_{j}\right| \log n+1+\sum_{k, l \neq i, j} \frac{16 \log n\left|v_{i}-v_{j}\right|^{2}}{A(k, l, i)} \quad \text { (by Lemmas } 3 \text { and } 6\right) \\
& =O\left(\left|v_{i}-v_{j}\right|^{2} \log ^{3} n\right) .
\end{aligned}
$$

The last step above follows from the constraint set (9) as follows:

$$
\sum_{k, l \neq i} \frac{1}{A(k, l, i)}=\sum_{k \neq i} \sum_{l \neq i, k} \frac{1}{A(i, k, l)}
$$

For each pair $i, k$ the inner sum is $O\left((\log n) /\left|v_{k}-v_{i}\right|\right)$. To see this, order the remaining vertices according to their distance from $i$ (say) and then the constraints imply that the triangle induced by the $p^{t h}$ point in this order has area at least $\Omega\left(p\left|v_{i}-v_{k}\right|\right)$. Hence

$$
\begin{aligned}
\sum_{k \neq i} \sum_{l \neq i, k} \frac{1}{A(i, k, l)} & \leq c \sum_{k \neq i} \frac{1}{\left|v_{i}-v_{k}\right|} \sum_{1 \leq p \leq n} \frac{1}{p} \\
& \leq c \log n \sum_{k \neq i} \frac{1}{\left|v_{i}-v_{k}\right|} \\
& =O\left(\log ^{2} n\right) .
\end{aligned}
$$

Here $c$ is a constant. The last step is implied by the constraint set (8).

Theorem 2 The expected length of the ordering found by the algorithm is $O\left(\log ^{2} n\right)$ times the optimum.

Proof. The expected value of the square of the length of the ordering found by our algorithm is

$$
\begin{aligned}
E\left(\sum_{(i, j) \in E} Y_{i j}^{2}\right) & =\sum_{(i, j) \in E} E\left(Y_{i j}^{2}\right) \\
& \leq \sum_{(i, j) \in E} O\left(\left|v_{i}-v_{j}\right|^{2} \log ^{3} n\right) \\
& \leq O\left(O P T_{S D P}^{2} \log ^{3} n\right) \\
& =O\left(O P T^{2} \log ^{4} n\right)
\end{aligned}
$$

where $O P T_{S D P}$ is the objective value of the SDP and hence (within a factor of $\sqrt{\log n}$ ) a lower bound on the minimum length of any linear ordering The result on the length of the ordering follows with high probability using Markov's inequality and taking square roots.

\section{How good is the SDP?}

What is the integrality gap of our first SDP? While our rounding procedure for the first SDP gives us an upper bound on the gap, it is possible that the gap is much smaller in 
reality. Note that our analysis is tight only for the specific rounding procedure we used, not the SDP itself.

Here we give some facts that indicate that the gap might be much smaller. One of the known lower bounds for the bandwidth of a graph is called the density lower bound [4]. It is defined as

$$
B_{d}=\max _{H} \frac{|H|-1}{\operatorname{diam}(H)},
$$

where the maximum is taken over all connected subgraphs of $G$.

It is interesting to note that the density lower bound is approximately computable (within a factor of 2) in polynomial time as follows: Imagine picking a center node in the subgraph $H^{*}$ achieving the bound, and consider the breadth-first tree rooted at this center truncated at level $\left\lceil\frac{\operatorname{diam}(H)}{2}\right\rceil$. The bound achieved by this subgraph is at least half the density lower bound. We can now search for the best such bound by looking over all choices of the root at all truncated breadth-first trees, for the best such subgraph.

The exact strength of the density lower bound is an open problem, but the largest known gap is $O(\log n)$ for an $n$-vertex graph. One of the known constructions of graphs which achieve this gap, the so-called Cantor combs, was described by Chung and Seymour $[3]$.

The following lemma says that the integrality gap of our simple relaxation is no larger than the gap between the density lower bound and the optimum.

Lemma 10 Let $(x, b)$ be the optimal solution of the bandwidth SDP. Then $b=\Omega\left(B_{d}\right)$.

Proof. Let $H$ be the subgraph of $G$ that achieves the maximum density. Since the average distance $d_{i j}$ between points in the solution corresponding to vertices of $H$ is $\Omega(|H|)$, there is a vertex $v$ of $H$ such that the total sum of distances between $x_{v}$ and the other points in $H$ is $\Omega\left(|H|^{2}\right)$. But this sum is at the same time at most $b|H| \operatorname{diam}(H)$, and so $b|H| \operatorname{diam}(H)=\Omega\left(|H|^{2}\right)$. That is,

$$
b=\Omega\left(\frac{|H|}{\operatorname{diam}(H)}\right) .
$$

The results of Feige [6] imply that the optimum bandwidth is at most a poly-logarithmic factor higher than the density bound, thus implying a similar integrality gap for our SDP formulation of the bandwidth problem. This leaves open the tantalizing possibility of better rounding schemes of the SDP solution to the problem.

\section{Conclusions and further work}

Along the lines of the constraint set (9), and Feige's result [6], it is possible to refine the semi-definite relaxation further (by using the spreading constraints on $k$-simplices instead of just edges and triangles). This yields poly-logarithmic approximations for any $L_{2 k}$ norm in $O\left(n^{2 k}\right)$ time and also a poly-logarithmic approximation for minimum bandwidth in quasipolynomial time $\left(n^{O(\log n)}\right)$ by considering subsets of size $\log n$. It is an open question as to whether we can solve this latter relaxation in polynomial time. 


\section{Acknowledgments}

We thank Seffi Naor for his thoughtful comments on an earlier draft of this paper, and for pointing out an error in the analysis of the minimum-length ordering guarantee in the preliminary version [1]. We also thank Egon Balas for comments that improved the readability of the paper.

\section{References}

[1] A. Blum, G. Konjevod, R. Ravi and S. Vempala, Semi-Definite Relaxations for Minimum Bandwidth and other Vertex-Ordering problems, Proceedings of the 30th Annual ACM Symposium on Theory of Computing, 90-99, 1998.

[2] P. Chinn, J. Chvátalová, A. Dewdney, N. Gibbs, The bandwidth problem for graphs and matrices-a survey, J. Graph Theory, 6: 223-254, 1982.

[3] F. R. K. Chung and P. D. Seymour, Graphs with small band- and cutwidth, Discrete Math. 75: 113-119, 1989.

[4] V. Chvátal, A remark on a problem of Harary, Czechoslovak Math J. 20: 109-111, 1970 .

[5] G. Even. J. Naor, S. Rao and B. Schieber, "Divide-and-conquer approximation algorithms via spreading metrics," Proceedings of the 35th Annual Conference on Foundations of Computer Science, 62-71, 1995.

[6] U. Feige, Approximating the bandwidth via volume respecting embeddings, Proceedings of the 30th Annual ACM Symposium on Theory of Computing, 90-99, 1998.

[7] M Garey, R. Graham, D. Johnson, D. E. Knuth, Complexity results for bandwidth minimization, SIAM J. Appl. Math. 34: 477-495, 1978.

[8] M. Grötschel, L. Lovász, A. Schrijver, Geometric Algorithms and Combinatorial Optimization, Springer, 1988.

[9] M. Goemans and D. Williamson, Improved approximation algorithms for maximum cut and satisfiability problems using semidefinite programming, JACM, 42: 1115-1145, 1995 .

[10] G. H. Golub and C. F. Van Loan, Matrix Computations. The Johns Hopkins University Press, Baltimore, MD, 1983.

[11] J. Haralambides, F. Makedon, B. Monien, Bandwidth minimization: an approximation algorithm for caterpillars, Math Systems Theory, 24:169-177, 1991.

[12] T. Kloks, D. Kratsch, H. Müller, Approximating the bandwidth for asteroidal triplefree graphs, Proceedings of ESA '95, LNCS 979, 434-447, Springer, 1995.

[13] B. Monien, The bandwidth-minimization problem for caterpillars with hairlength 3 is NP-complete, SIAM J. Algebraic Discrete Methods, 7: 505-512, 1986. 
[14] Y. Nesterov and A. Nemirovskii, Interior-point polynomial algorithms in convex programming, SIAM Studies in Applied Math. Vol. 13 (1994).

[15] C. H. Papadimitriou, The NP-completeness of the bandwidth minimization problem, Computing, 16: 263-270, 1976.

[16] J. Naor, personal communication, July 1998.

\section{Appendix}

Lemma 11 If $u_{1}, \ldots, u_{n}$ are the points in the $(\lfloor\log n\rfloor+1)$-dimensional space defined in the proof of lemma 8, there is a constant $c \geq 0.02$ such that $A(i, j, k) \geq c(j-i) \cdot(k-j)$ for all $i<j<k$.

Proof. The idea of the proof is as follows: for a triangle defined by $u_{i}, u_{j}$ and $u_{k}$, we consider its projection on a two-dimensional plane $P_{\ell}$ spanned by the coordinate vectors $e_{1}$ and $e_{\ell}$, for different values of $\ell$. Clearly, the area of each such projection is a lower bound on the area of the original triangle. The area of a triangle can be calculated as $\frac{1}{2} a b \sin \phi$ where $a$ and $b$ are two sides of the triangle and $\phi$ is the angle between them. If $u_{i}^{\prime} u_{j}^{\prime} u_{k}^{\prime}$ is the projection of $u_{i} u_{j} u_{k}$ onto $P_{\ell}$, then $\left|u_{i}^{\prime}-u_{j}^{\prime}\right| \geq(j-i)$ and $\left|u_{j}^{\prime}-u_{k}^{\prime}\right| \geq(k-j)$. Thus, if we can show that for each triple $i, j, k$ there exists a coordinate $\ell$ such that the angle at $u_{j}^{\prime}$ (the projection of $u_{j}$ onto $P_{\ell}$ ) is bounded above by some universal constant $\phi$, we will be done. In what follows we use an inductive case analysis to show that we can always ensure $\phi<177.5^{\circ}(\sin \phi \geq 0.04)$.

We assume without loss of generality that $j \leq n / 2$ and $k>n / 2$. (If $k \leq n / 2$ or $i \geq n / 2$ then we can work with $n / 2$ instead of $n$ and the claim holds by induction. The two cases, $j \geq n / 2$ and $j \leq n / 2$ are the same by symmetry so we only work with the first one.) If $k \geq 9 n / 16$ then after projecting to $P_{2}$, the angle at $u_{j}^{\prime}$ is at most $\sin ^{-1}(1 / 8)<173^{\circ}$, so we can assume $n / 2<k<9 n / 16$.

If $j \leq n / 4$, then projecting onto $P_{3}$ works since the slope of the line through $u_{j}^{\prime}$ and $u_{k}^{\prime}$ is at most $1 / 16$, so the angle at $u_{j}^{\prime}$ is roughly $135^{\circ}$ (precisely, the angle is at most $\left.135^{\circ}+\tan ^{-1}(1 / 9)<142^{\circ}\right)$. If $i \geq n / 4$ the claim holds by induction.

Now there are four cases left.

1. If $i \leq n / 8$ and $n / 4 \leq j \leq 3 n / 8$, then the angle at $u_{j}^{\prime}$ in $P_{3}$ is at most $180^{\circ}-$ $\tan ^{-1}(1 / 3)<162^{\circ}$.

2. If $n / 8 \leq i \leq n / 4$ and $n / 4 \leq j \leq 3 n / 8$ we consider two subcases:

(a) $(j-n / 4) \leq 5 / 3(n / 4-i)$ : Project onto $P_{3}$. The slope of the line through $u_{i}^{\prime}$ and $u_{j}^{\prime}$ is $\geq-1 / 4$ and the slope of the line through $u_{j}^{\prime}$ and $u_{k}^{\prime}$ is $\leq-1 / 3$. So, the angle at $u_{j}^{\prime}$ is at most $176^{\circ}$.

(b) $(j-n / 4) \geq 5 / 3(n / 4-i)$ : Project onto $P_{4}$. The slope of the line through $u_{i}^{\prime}$ and $u_{j}^{\prime}$ is $\geq 1 / 4$ and the slope of the line through $u_{j}^{\prime}$ and $u_{k}^{\prime}$ is $\leq 1 / 5$, so the angle at $u_{j}^{\prime}$ is at most $177.5^{\circ}$. 
3. If $i \leq n / 8$ and $3 n / 8 \leq j \leq n / 2$, then note that the slope in $P_{3}$ of the line between $u_{i}^{\prime}$ and $u_{j}^{\prime}$ is $\geq-1 / 3$. So, if the slope of the line through $u_{j}^{\prime}$ and $u_{k}^{\prime}$ in $P_{3}$ is $\leq-1 / 2$ then the angle at $u_{j}^{\prime} \leq 172^{\circ}$. Otherwise, it must be the case that the slope of the line through $u_{j}^{\prime}$ and $u_{k}^{\prime}$ in $P_{2}$ is $\leq 1 / 2$ (and the slope of the line through $u_{i}^{\prime}$ and $u_{j}^{\prime}$ in $P_{2}$ is 1$)$, so in $P_{2}$ the angle at $u_{j}^{\prime} \leq 162^{\circ}$.

4. Finally, if $n / 8 \leq i \leq n / 4$ and $3 n / 8 \leq j \leq n / 2$ then this is analogous to the previous case but using $P_{4}$ instead of $P_{3}$. In other words, in $P_{4}$, the slope of the line between $u_{i}^{\prime}$ and $u_{j}^{\prime}$ is $\geq-1 / 3$. So, if the slope of the line through $u_{j}^{\prime}$ and $u_{k}^{\prime}$ in $P_{4}$ is $\leq-1 / 2$ then the angle at $u_{j}^{\prime} \leq 172^{\circ}$. Otherwise the slope of the line in $P_{2}$ between $u_{j}^{\prime}$ and $u_{k}^{\prime}$ must be $\leq 1 / 2$ so in $P_{2}$ the angle at $u_{j}^{\prime} \leq 162^{\circ}$.

This research was sponsored in part by National Science Foundation (NSF) grant no. CCR-0122581. 